\title{
Health-Care and Societal Costs Associated with Non- Persistence with Subcutaneous TNF- $\alpha$ Inhibitors in the Treatment of Inflammatory Arthritis (IA): A Retrospective Observational Study
}

\author{
Johan Dalén · Anushri Chitkara • Axel Svedbom • Tor Olofsson • \\ Amy Puenpatom · Christopher M. Black · Zaina P. Qureshi (D)
}

Received: August 30, 2021 / Accepted: October 18, 2021 / Published online: November 9, 2021

(C) The Author(s) 2021

\begin{abstract}
Objective: A few studies have suggested that patients with inflammatory arthritis (IA) who remain persistent with subcutaneous TNF- $\alpha$ inhibitors (SC-TNFi) incur lower health care costs than patients who discontinue treatment, whereas data on the impact of non-persistence on indirect costs
\end{abstract}

Digital Features This article is published with digital features, including an infographic, to facilitate understanding of the article. To view digital features for this article go to https://doi.org/10.6084/m9.figshare. 17136773.

Supplementary Information The online version contains supplementary material available at https:// doi.org/10.1007/s12325-021-01970-w.

J. Dalén · A. Svedbom

ICON Plc, Stockholm, Sweden

A. Chitkara

ICON Plc, Bangalore, India

T. Olofsson

Department of Clinical Sciences Lund,

Rheumatology, Lund University, Skåne University

Hospital, Lund, Sweden

A. Puenpatom · C. M. Black · Z. P. Qureshi ( $\square)$ Center for Observational and Real-World Evidence (CORE), Merck \& Co., Inc., 2000 Galloping Hill Rd, Kenilworth, NJ 07331, USA

e-mail: zaina.qureshi@merck.com are largely lacking. Furthermore, existing estimates are based on fixed follow-ups, in relation to treatment initiation, and therefore do not measure costs in direct relation to treatment discontinuation. Therefore, by capturing costs in direct relation to treatment discontinuation, this study aimed to estimate direct and indirect costs associated with non-persistence with SC-TNFis in IA.

Methods: Adult Swedish biologic-naïve IA patients initiating biologic treatment with a SCTNFi (adalimumab, etanercept, certolizumab or golimumab) between May 6, 2010, and December 31,2017 , were identified in population-based registers with almost complete coverage. IA was defined as a diagnosis of rheumatic arthritis, ankylosing spondylitis/unspecified spondyloarthritis or psoriatic arthritis. Non-persistent patients were matched on propensity score to patients persistent with treatment by at least an additional 12 months. This enabled comparisons of direct healthcare costs and indirect costs for sick leave and disability pension, respectively, 12 months before and 12 months after treatment discontinuation. 
Results: A balanced cohort of 486 matched pairs was generated. The total direct and indirect costs were significantly higher among non-persistent patients already during the 12 months before index $(\$ 20,802[18,335-23,429]$ vs. $\$ 16,600$ [14,331-18,696]). However, while non-persistent patients increased their total direct and indirect costs, persistent patients significantly decreased the same, further widening the difference in costs during the 12-month period after index date
$(\$ 22,161 \quad[19,754-24,556] \quad$ vs. $\quad \$ 13,465$ [11,415-15,729]).

Conclusions: Among biologic-naïve Swedish IA patients treated with SC-TNFis, persistent patients incurred about $40 \%$ lower aggregated direct and indirect costs compared to non-persistent patients the year following SC-TNFi discontinuation. This highlights the impact of treatment persistence from an economic viewpoint, adding further aspects to the clinical perspective.

Graphical Abstract: 
HEALTH-CARE AND SOCIETAL COSTS ASSOCIATED WITH NON-PERSISTENCE WITH SUBCUTANEOUS (SC) TNF- $\alpha$ INHIBITORS FOR INFLAMMATORY ARTHRITIS (IA)

Johan Dalén, Anushri Chitkara, Axel Svedbom, Tor Olofsson, Amy Puenpatom, Christopher M. Black, Zaina P. Qureshi

A RETROSPECTIVE, OBSERVATIONAL STUDY USING SWEDISH HEALTH DATA REGISTERS

\section{PATIENTS: \\ هั}

Biologic-naïve patients in Sweden who received SC-TNFi treatment for IA and remained eligible after applying the inclusion criteria.

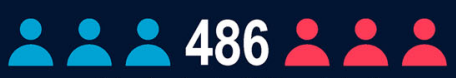

A propensity score matched cohort of 486 matched pairs was generated as persistent and non-persistent patients.

Persistence: Time from treatment initiation to treatment discontinuation or switch to another SC-TNFi or bDMARD

PERSISTENCE

TOTAL DIRECT AND INDIRECT COSTS PRE-INDEX DATE $\$ 16,600$ $[95 \% \mathrm{Cl}, 14,331-18,690$

TOTAL DIRECT AND INDIRECT COSTS POST-INDEX DATE*

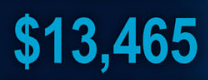

$[95 \% \mathrm{Cl}, 11,415-15,729]$

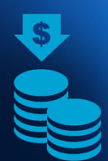

$n=486$

NON-PERSISTENCE

Total direct and indirect costs were significantly higher

among non-persistent patients 12 months pre-index date.

Among biologic-naïve patients in Sweden treated with SC-TNFi for IA, persistent patients incurred $\sim 40 \%$ lower aggregated direct and indirect costs than non-persistent patients 12 months post-index date.

Persistent patients significantly decreased total direct and indirect costs 12 months post-index date.
TOTAL DIRECT AND INDIRECT COSTS PRE-INDEX DATE

$\$ 20,802$

[95\% Cl, 18,335-23,429]

*Please refer to the journal article for a full analysis of persistent and non-persistent cohort data.

bDMARD = biological disease-modifying antirheumatic drug; $\mid \mathrm{A}=$ inflammatory arthritis; SC-TNFi = subcutaneous tumor necrosis factor inhibitor. 
Keywords: Biologics; Tumor necrosis factoralpha inhibitors; Treatment persistence; Rheumatoid arthritis; Psoriatic arthritis; Ankylosing spondylitis; Spondyloarthritis

\section{Key Summary Points}

A handful of studies have shown that nonpersistence in the treatment with SCTNFis in IA is associated with higher health care costs, but data on the impact on indirect costs are largely lacking.

Existing estimates of cost impact are based on fixed follow-up in relation to treatment discontinuation and therefore do not measure costs in direct relation to treatment discontinuation.

Capturing costs in direct relation to treatment discontinuation, this study aimed to estimate direct and indirect costs associated with non-persistence with SCTNFis in IA.

Based on high-quality data from Swedish administrative registers, this study showed that non-persistence in first-line treatment with SC-TNFis in IA was associated with higher costs. While nonpersistent patients increased their health care costs from the year before to the year after treatment discontinuation, persistent patients significantly decreased their costs pertaining to health care and sick leave over the same time period.

The results of this study highlight the impact of treatment persistence from an economic viewpoint, adding further aspects to the clinical perspective.

\section{INTRODUCTION}

Inflammatory arthritis (IA) is a group of inflammatory joint diseases where the most common are rheumatoid arthritis (RA), ankylosing spondylitis (AS) and psoriatic arthritis (PsA), presenting as painful, swollen and stiff peripheral joints (in RA and PsA) and/ or axial joints including the sacroiliac joints (as in AS and sometimes in PsA) $[1,2]$. These diseases are characterized by elevated levels of inflammatory markers, including cytokines such as tumor necrosis factor- $\alpha$ (TNF- $\alpha)$. Since IA is not curable, treatment efforts are aimed at minimizing associated disability and maximizing health-related quality of life by controlling inflammation and disease symptoms, preventing progressive structural joint damage, and preserving or normalizing joint function [3-5].

Disease management in IA has transformed over the recent decades with the addition of biologic disease-modifying anti-rheumatic drugs (bDMARDs), including subcutaneous TNF- $\alpha$ inhibitors (SC-TNFi) [6]. Apart from being a surrogate marker for clinical effectiveness, safety and treatment satisfaction in IA [7-9], treatment persistence with SC-TNFis has also been associated with significant cost offsets in health-care resource utilization (HCRU) [10-12]. While likely to have similar impact on indirect costs, e.g., costs of absenteeism due to sick leave and early retirement (from sickness or injury [i.e., disability pension]), evidence thereof is scarce. Although a recent German study [13] using claims data has assessed the association between indirect costs and nonpersistence with SC-TNFis in IA, populationbased data sources are yet to be leveraged. Furthermore, most previous studies, including the German study, have assessed costs associated with non-persistence over a fixed follow-up period from the date of treatment initiation. To the best of our knowledge, no study to date has captured costs over an interval around (i.e., before and after) treatment discontinuation, where persistent and non-persistent patients were matched on patient characteristics at treatment initiation and duration of first-line treatment, thus accounting for differences which may otherwise bias estimates of costs attributable to non-persistence.

By employing this approach to analysis and utilizing population-based Swedish registries with almost complete coverage, this study aimed to estimate direct and indirect costs 
associated with non-persistence with SC-TNFis in IA.

\section{METHODS}

\section{Data Sources}

Six data sources were used for this retrospective observational study, of which three were National Health Data Registers (NHDRs) held by the National Board of Health and Welfare (NBHW) in Sweden. With further details outlined elsewhere [10], the Prescribed Drug Register (PDR) covers all prescriptions filled outside of the hospital setting in Sweden since July 1, 2005, while the National Patient Register (NPR) contains information on both inpatient care and specialized outpatient care since 2001. The Cause of Death Register (CDR), data which were used to determine patient vital status throughout the study, records all deaths in Sweden including the underlying cause.

Complementary information on indirect costs in IA was collected from the MikroData för Analys av Socialförsäkringen (MiDAS) register held by the Swedish Social Insurance Agency (Försäkringskassan). The register contains daylevel, social insurance data on an individual basis, covering sick leave, disability pension and rehabilitation. Since no population-based register in Sweden keeps and maintains data on primary care on a national level, this information was extracted from a regional register covering the county of Västra Götaland, which is the second largest county in Sweden and accounts for around $17 \%$ of the total Swedish population. Consequently, this study was restricted to Swedish IA patients residing in this region. Lastly, information on educational level of the patients, primarily for minimizing confounding, was extracted from Statistics Sweden.

Information from each register was linked by NBHW using a unique personal identification number assigned for each Swedish citizen. The authors of this study had no access to the personal identification numbers and did not participate in the database linkage process. Ethical approval for this study was obtained from an Ethics Review Board in Sweden (2019-02774).

\section{Study Design and Patient Selection}

Figure 1 presents an overview of the study design. Inclusion and exclusion criteria were implemented to identify biologic-naïve adult Swedish IA patients starting biologic therapy with a SC-TNFi. Eligible candidates for inclusion had to initiate treatment between May 6, 2010 (date of inclusion of all currently available SCTNFis in the Swedish reimbursement system), and December 31, 2017, with a SC-TNFi (adalimumab, certolizumab pegol, etanercept, or golimumab) [14, 15]. Treatment initiation was defined as the date of the first filled prescription, and a 'wash-out' period (i.e., no prescription filled between July 1, 2005, and May 6, 2010) was applied. Patients were excluded if they (1) were $<18$ years of age at treatment initiation; (2) had records of a TNFi administered in an in- or outpatient hospital setting before the date of treatment initiation; (3) had < 12 months of baseline and follow-up periods as a result of emigration, death, or end of data availability; (4) had a prescription of a SC-TNFi filled from any department apart from rheumatology, orthopedics or rehabilitation, or a prescription filled by a non-rheumatologist; and/or (5) had no record of an IA diagnosis as defined in this study (i.e., AS/unspecified spondyloarthritis [ICD10: M08.1, M45, M46.1, M46.8 and M46.9], PsA [ICD-10: L40.5, M07.0, M07.1, M07.2 and M07.3] or RA [ICD10: M05.8, M05.9, M06.0, M06.9 and M12.3]). The second exclusion criterion was aimed at curtailing the risk of deriving incorrect estimates of treatment duration as a result of misleading gaps in the PDR data related to patients being administered prior TNFi therapy in an in- or outpatient hospital setting. However, these gaps are anticipated to be minimal for SC-TNFi treatments as the majority $(99 \%)$ of SC-TNFi prescriptions are made available and handed out by pharmacies [16]. Additionally, the second criterion also ensured the exclusion of patients administered biologics via intravenous route in a hospital setting, thus further ascertaining that included patients were biologics-naïve. Lastly, to exclude patients not responding to induction treatment, only those patients reaching the maintenance period (defined to occur after 6 months of 


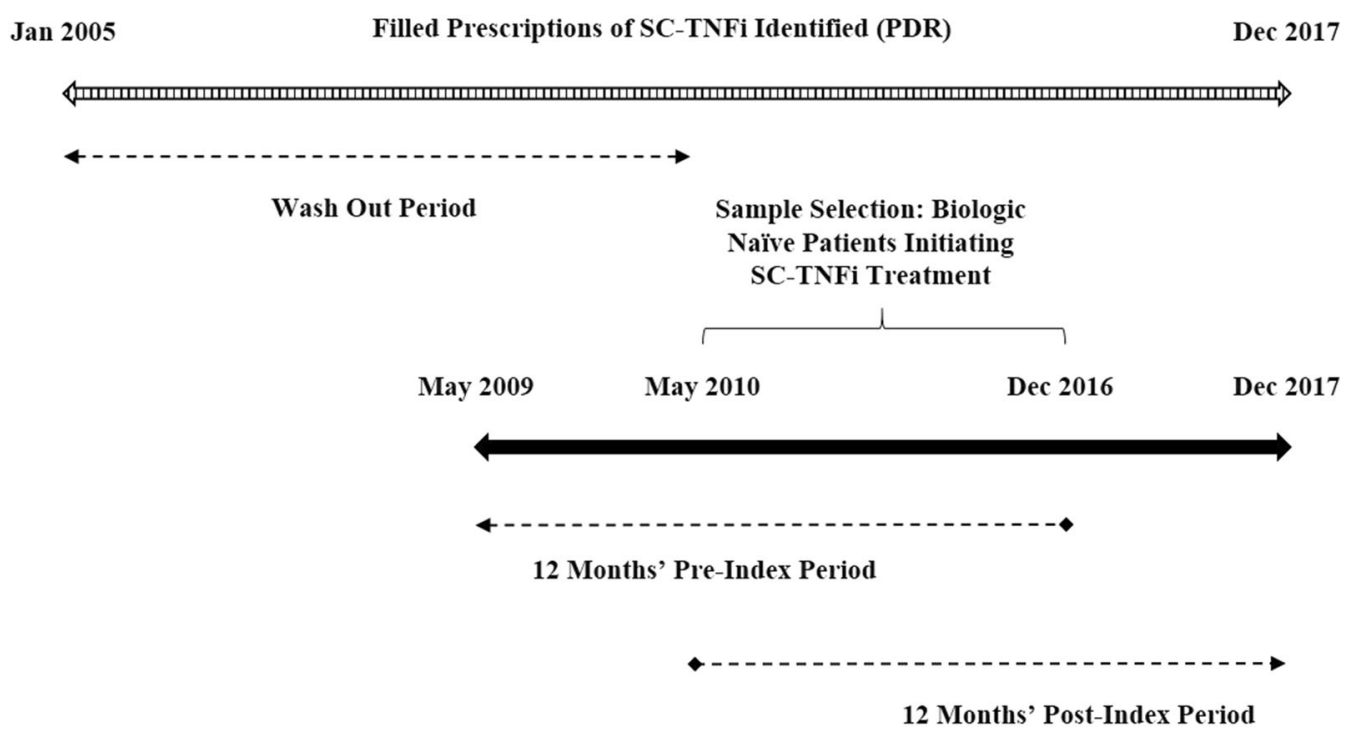

Fig. 1 Study design

continuous first-line treatment) before being deemed non-persistent were considered.

\section{Stratification and Matching}

To enable comparisons and to assess the direct and indirect costs associated with non-persistence, patients were stratified as persistent and non-persistent patients based on persistence status at 24 months after SC-TNFi treatment initiation. Applying the definition of the Medication Compliance Work Group at the International Society for Pharmacoeconomics and Outcomes Research (ISPOR) [17], persistence was derived, using prescription information from the PDR, as the time elapsed between treatment initiation and treatment discontinuation. The number of packages dispensed was multiplied by the defined daily dose (DDD), while allowing for a 60-day grace period between prescriptions. Patients who discontinued treatment or switched to either a second SC-TNFi or another bDMARD were categorized as "non-persistent."

Due to the observational nature of the study, non-persistent patients (cases) were matched on propensity score to persistent patients (controls) to mitigate potential selection bias. The propensity score was based on the patient characteristics presented in Table 1, including co-medication, comorbidities and education level as well as occurrence of any record of sick leave from 6 months before to 6 months after treatment initiation. In addition, to facilitate comparisons across the same time period following the initiation of first-line SC-TNFi treatment, the propensity score-matched (PSM) cohort was derived in sequential steps. Nonpersistent patients were grouped according to retention of first-line treatment in three 6-month intervals (i.e., 6-12 months, 12-18 months and 18-24 months) and were then matched to persistent controls who continued their first-line treatment by at least an additional 12 months, until no more matches could be made. The index date for this study was defined as the date of non-persistence and was derived from the number of days from treatment initiation to treatment discontinuation for cases and assigned to both cases and their matched controls, as depicted in Fig. 2. This ensured the same index date for the matched cases and controls and thereby the same followup period in relation to treatment initiation.

\section{Study Outcomes}

Costs associated with non-persistence with SCTNFis treatment in IA were operationalized and assessed as the cost of HCRU and absenteeism 
Table 1 Baseline characteristics of propensity score-matched cohort

\begin{tabular}{|c|c|c|c|}
\hline & Non-persistent patients, $n=486$ & Persistent patients, $n=486$ & $p$ value \\
\hline Age-mean $(S D)$ & $48.16(15.11)$ & $48.93(15.31)$ & 0.422 \\
\hline Female- $n(\%)$ & $308(63.4)$ & $308(63.4)$ & 1.000 \\
\hline Diagnosis-n (\%) & & & 0.685 \\
\hline PsA & $91(18.7)$ & $101(20.8)$ & \\
\hline $\mathrm{AS} / \mathrm{uSpA}$ & $128(26.3)$ & $129(26.5)$ & \\
\hline RA & $267(54.9)$ & $256(52.7)$ & \\
\hline $\mathrm{CCI}-$ mean $(\mathrm{SD})$ & $1.47(1.37)$ & $1.55(1.34)$ & 0.289 \\
\hline \multicolumn{4}{|l|}{ Co-medication-n $(\%)$} \\
\hline NSAIDs & $377(77.6)$ & $367(75.5)$ & 0.449 \\
\hline csDMARDs & $362(74.5)$ & $362(74.5)$ & 1.000 \\
\hline Steroids & $265(54.5)$ & $254(52.3)$ & 0.479 \\
\hline Outpatient visits-mean (SD) & $6.51(4.88)$ & $6.47(4.99)$ & 0.776 \\
\hline Inpatient stays-mean (SD) & $0.32(0.89)$ & $0.32(0.87)$ & 0.999 \\
\hline Level of education & & & 0.533 \\
\hline Compulsory school & $91(18.7)$ & $82(16.9)$ & \\
\hline Upper secondary/high school & $217(44.7)$ & $224(46.1)$ & \\
\hline Post upper secondary edu. $<3$ years & $65(13.4)$ & $75(15.4)$ & \\
\hline Post upper secondary edu. $\geq 3$ years & $111(22.8)$ & $100(20.6)$ & \\
\hline Postgraduate studies & $2(0.4)$ & $5(1.0)$ & \\
\hline
\end{tabular}

In addition to standardized differences in covariates after matching, differences in baseline characteristics were also assessed with the non-parametric Wilcoxon-Mann-Whitney test and the chi-square test for interval and discrete outcomes, respectively

$P s A$ psoriatic arthritis, $A S / u S p A$ ankylosing spondylitis/unspecified spondyloarthritis, $R A$ rheumatic arthritis, $C C I$ Charlson Comorbidity Index, $c s D M A R D s$ conventional synthetic disease-modifying anti-rheumatic drugs, NSAIDs non-steroidal antiinflammatory drugs, $S D$ standard deviation

(i.e., due to sick leave and disability pension) and captured continuously by month as well as accumulated during the 12-month periods before and after the index date. HCRU comprised outpatient care, specialized care provided by doctors and other health care practitioners in a hospital setting as well as primary care, inpatient stays and non-bDMARD medication. Indirect costs, captured as productivity losses due to sick leave and disability pension, were estimated by the human capital approach and thus based on the mean daily salary plus employer fees and social security contributions paid by the employer. The average monthly salary in 2018 for the working population in Sweden was 34,600 SEK (USD 3984) and the employer's social security contribution was $31.42 \%$ of the total salary [18].

The development of monthly continuous costs, measured over the 24-month period around the index date, was studied descriptively only. The accumulated aggregate costs for 


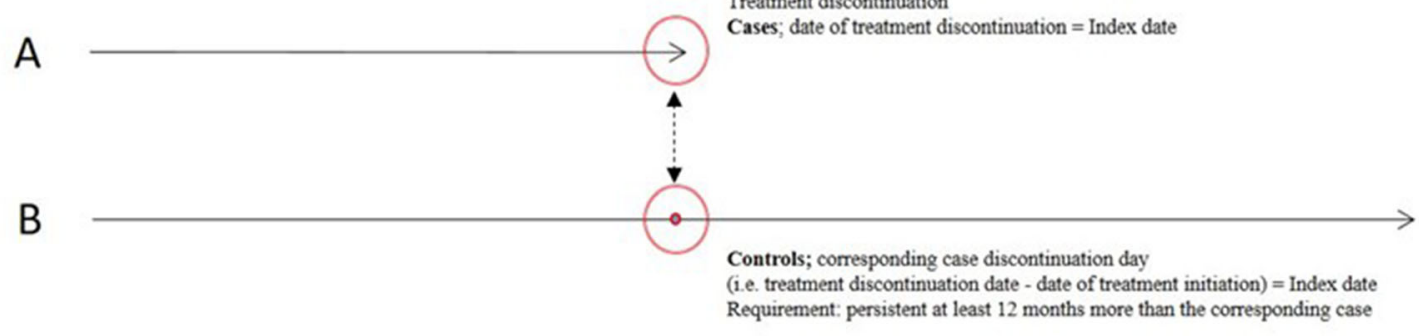

12 Month Pre Index Period

12 Month Post Index Period

Fig. 2 Index date defined as treatment discontinuation

persistent and non-persistent patients were compared for differences within (pre- vs. postindex costs) as well as across the two groups. All costs were presented in year 2018 US dollars (USD; \$). The included patients were further described using patient characteristics captured over a 12-month period prior to SC-TNFi treatment initiation including, e.g., age, gender, IA diagnosis, use of steroids, non-steroidal antiinflammatory drugs (NSAIDs) and conventional systemic DMARDs (csDMARDs), and highest level of education.

\section{Statistical Analysis}

A logit model was used to derive the propensity score required to generate the PSM cohort. A Greedy algorithm (i.e., once a patient has been matched they cannot be considered for another match) with 5-1 digit matching was used to generate matched pairs. Standardized differences in covariates before and after matching were compared to assess balance across strata (defined as standardized differences $<0.1$ by Rosenbaum and Rubin [19]) [20-22]. Additionally, baseline characteristics were compared using non-parametric
Wilcoxon-Mann-Whitney tests and chi-square tests, for interval and discrete outcomes, respectively. The distributional character of aggregated cost components collected 12 months before and after index date was assessed with histograms. Due to the generally non-symmetrical and skewed nature of HCRU and indirect costs [23], estimates of means and standard deviations (SD) were presented together with bootstrapped 95\% confidence intervals (CI) based on unrestricted random sampling with replacement and confidence limits derived from percentiles. Similarly, cost comparisons across patients were based on bootstrapped CIs for differences in group means, where significance was determined based on whether the interval included zero. To assess the robustness of the presented estimates, a number of sensitivity analyses were performed. First, given the observational nature of the study and inherent risk for unobserved confounders, the e-value (i.e., the strength, on the risk ratio scale that a confounding factor would need to have to explain away subgroupoutcome associations) was estimated in accordance with Mathur et al. and VanderWeele et al. $[24,25]$ for the standardized difference in mean 
total direct and indirect costs before and after index date. Second, bias-corrected and accelerated 95\% CIs were derived to assess the confidence limits of the 95\% CIs derived using the percentile method and are presented in Table 2. Third, differences in costs across persistent and non-persistent patients were also evaluated using generalized linear models (GLMs) with a log-link and gamma distributed error terms. The analyses of this study were conducted using SAS $₫$ software, version 9.4 of the SAS system for Windows, Copyright $\odot$ 2016, SAS Institute Inc., Cary, NC, USA.

\section{RESULTS}

\section{Study Population and Patient Characteristics}

Figure 3 illustrates the sequential patient selection. A total of 27,006 biologic-naïve adult IA patients initiating treatment with a SC-TNFi between May 1, 2010, and December 31, 2017, were identified. After application of the inclusion and exclusion criteria, 2374 eligible patients from the county of Västra Götaland remained. Of the 1530 patients who reached the maintenance period, a successfully balanced cohort of 486 matched pairs could be generated as persistent and non-persistent patients.

Patient characteristics at treatment initiation are summarized in Table 1 . Overall, the mean age was around 49 years, and almost two-thirds of the included patients were women. More than half were diagnosed with RA, while AS/ unspecified spondyloarthritis and PsA accounted for roughly a quarter and a fifth of the patients, respectively. Around half of the patients had used steroids while three quarters had used NSAIDs and csDMARDs during the year prior to SC-TNFi treatment initiation. The average number of outpatient visits was 6.5 , and the mean number of hospitalizations was approximately 0.3 . Most of the cohort had compulsory school or upper secondary school as their highest level of education.

\section{Health Care Resource Utilization (HCRU) and Indirect Costs}

The development of direct (HCRU) costs (excluding bDMARDs) and costs of sick leave and disability pension in persistent and non-persistent patients over the 12-month periods before and after the index date are presented in Fig. 4a-f. The monthly mean outpatient care costs for persistent and non-persistent patients were similar until about 5 months prior to the index date. Thereafter, the costs for non-persistent patients increased, exceeding $\$ 400$ around the index date and stabilizing at roughly $\$ 300$ per month. During the same period, persistent patients decreased their costs for outpatient care, which stabilized at around $\$ 200$ postindex date (Fig. 4a). Similarly, the monthly mean cost of inpatient care was comparable in the pre-index period, after which non-persistent patients increased their inpatient care costs through the remainder of follow-up, with substantially higher monthly average mean inpatient cost estimates than persistent patients (Fig. 4b). The costs for non-biologic medication in the two groups closely approximated each other throughout the entire follow-up period at $<\$ 50$ per month (Fig. 4c). The development of total HCRU costs across the 24 months followed the development of out- and inpatient care (Fig. 4d). While being similar between the groups in the pre-index period, indirect costs due to sick leave (Fig. 4e) were consistently higher among non-persistent patients compared to persistent patients for the rest of the follow-up period. Conversely, although consistently higher in non-persistent patients, the costs of disability pension (Fig. 4f) were stable across persistent and non-persistent patients across the 24 months of follow-up. Hence, the widening gap in total indirect costs between the two groups during the post-index period was primarily owing to a decrease in sick leave costs for persistent patients.

Histograms showed that the HCRU (direct) and indirect costs accumulated over 12 months before and after the index date were non-symmetrical, rightskewed and approximately gamma distributed. Table 2 presents the mean and standard deviation together with 


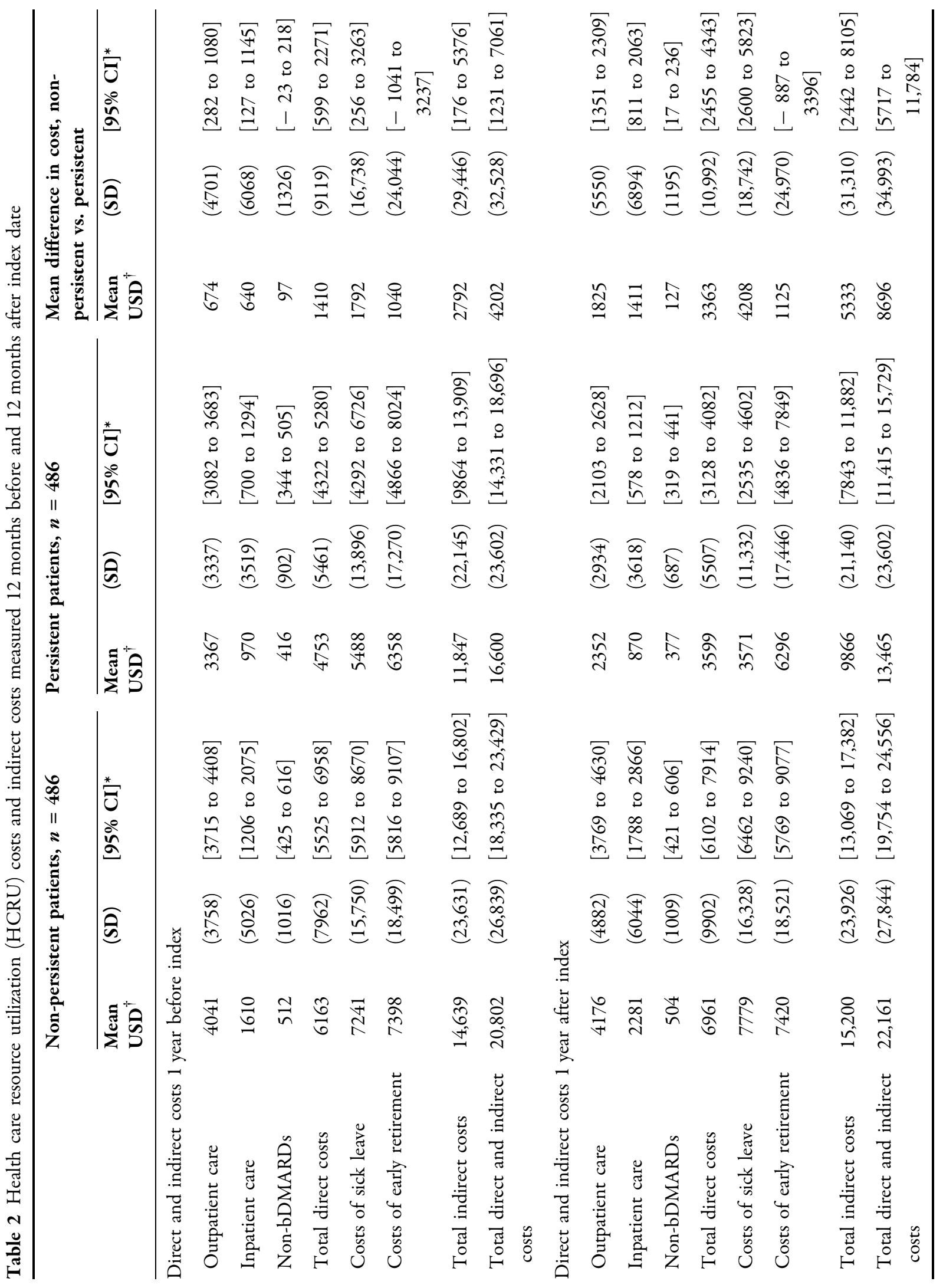




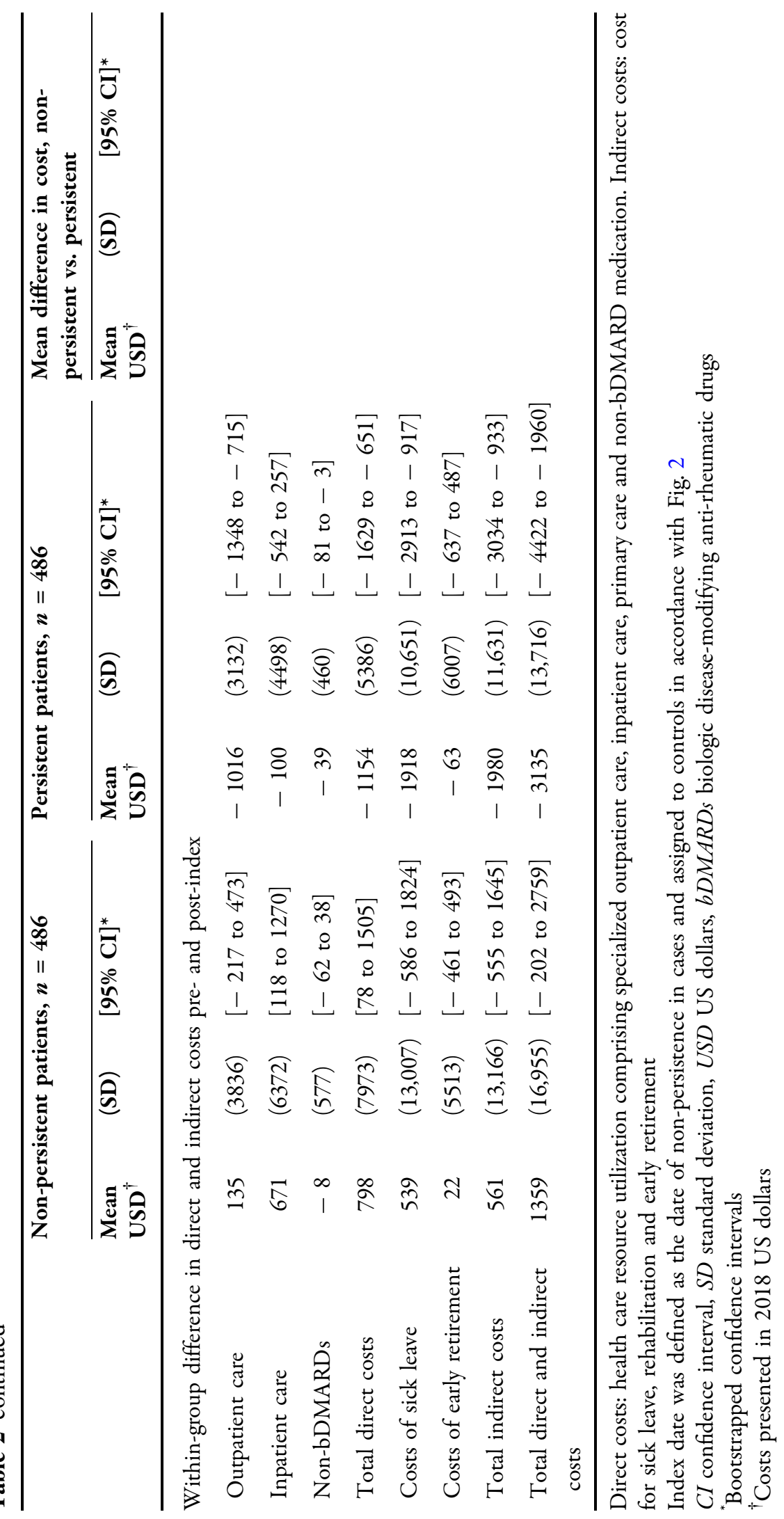




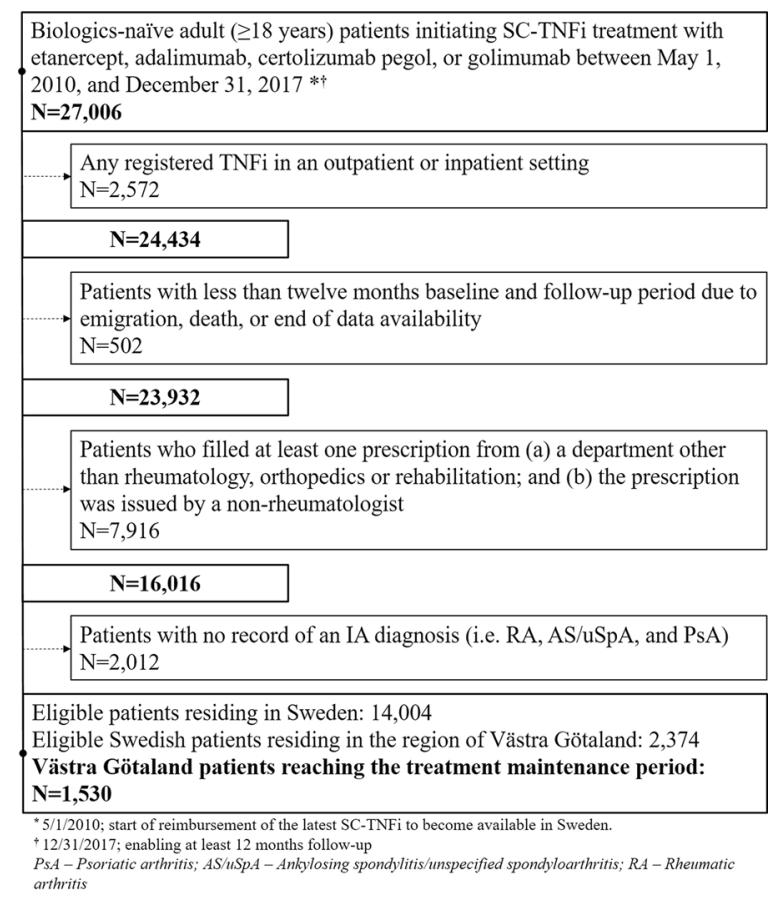

Fig. 3 Patient selection flow chart

bootstrapped confidence intervals for the total aggregate costs accumulated over a period of 12 months before and 12 months after the index date, stratified by persistent and nonpersistent patients. In the pre-index period, while the costs for non-bDMARD medication were observed to be comparable between persistent and non-persistent groups, the costs for out- and inpatient care were significantly lower among persistent patients, making for a significant difference in mean total cost for HCRU (\$4753; 95\% CI [4322-5280] for persistent patients vs. \$6163 [5525-6958] for non-persistent patients). However, while persistent patients lowered their total costs of HCRU between pre- and post-index years $(\$-1154$ [- 1629 to -651$])$, non-persistent patients increased their costs in the same time $\$ \mathbf{~} 798$ [78-1505]), thereby further broadening the cost gap. While this increase in the HRCU cost gap was primarily attributed to a significant decrease in outpatient care costs among persistent patients $(\$-1016[-1348$ to -715$])$, there was also a significant increase in the inpatient costs for non-persistent patients (\$671 [118-1270]). The total indirect costs due to sick leave and disability pension also differed significantly between persistent and non-persistent patients in the pre-index period $(\$ 11,847$ [9864-13,909] vs. $\$ 14,639$ [12,689-16,802]). However, a significant decline of $\$-1980$ ( -3034 to -933$)$ was observed in total indirect costs incurred by persistent patients between the pre- and post-index years, predominantly owing to a drop in the costs of sick leave, which further widened the difference in indirect costs between the groups in the year following treatment discontinuation.

Aggregated, the pattern was the same and the combined total direct and indirect costs were significantly higher in non-persistent patients already during the 12 months before index date $(\$ 20,802 \quad[18,335-23,429]$ vs. $\$ 16,600$ [14,331-18,696]). While non-persistent patients increased their total aggregated costs, persistent patients significantly decreased the same, further widening the difference in costs, making for a more pronounced, significant between-group difference for the 12-month period after index date $(\$ 22,161$ $[19,754-24,556]$ vs. $\$ 13,465[11,415-15,729])$.

Additionally, as displayed in supplementary Figure S1a-f, the results appear similar across all indications, with lower direct and indirect costs in persistent patients and with the largest visual difference observed in RA.

Three sensitivity analyses were performed to assess the robustness of the findings in IA patients overall. First, we derived the e-values for the observed standardized differences in mean total direct and indirect costs before and after index date. Given the covariates included in the PSM, these analyses showed that the strength of an unobserved confounding factor needed to explain away these subgroup-outcome associations was $\sim 1.6$ and $\sim 2.0$, respectively, on the risk ratio scale. Second, bias corrected and accelerated 95\% CIs, derived for total cost of HCRU, total indirect costs and the total cost of HCRU and indirect costs, before and after index date, were consistent with those derived using the percentile method, presented in Table 2. Similarly, the GLMs fitted to the same cost components, with persistence status as explanatory variable, were for all comparisons consistent with the bootstrapped mean differences across persistent and non-persistent patients. 

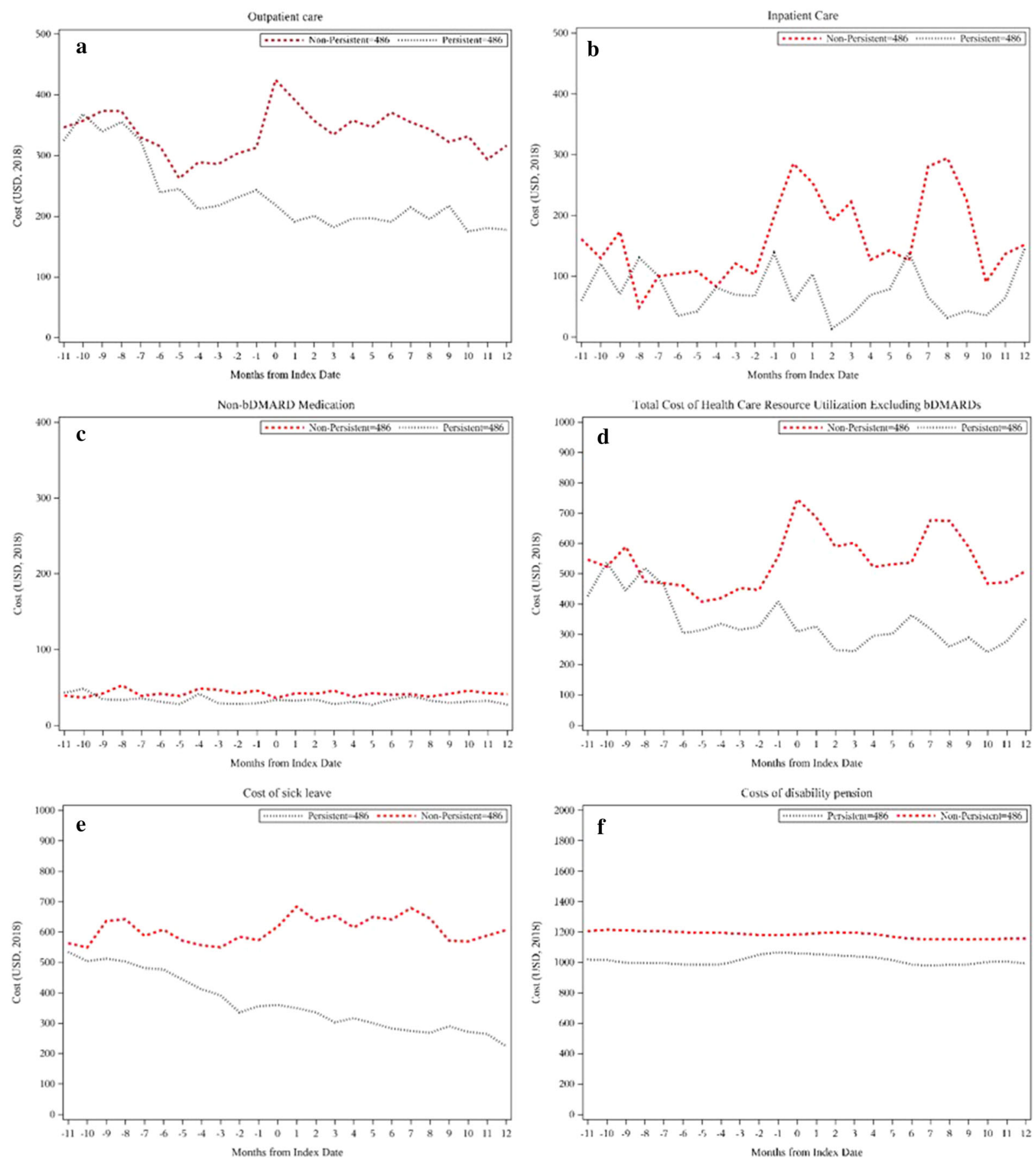

Fig. 4 Development of patient costs, from 12 months before to 12 months after index date, by persistence status. a Outpatient care, b inpatient care, $\mathbf{c}$ non-bDMARD medication, d total HCRU costs excluding bDMARDs,

e sick leave, $\mathbf{f}$ disability pension. Index date was defined as the date of non-persistence in cases and assigned to controls in accordance with Fig. 2 


\section{DISCUSSION}

Based on high-quality data from Swedish administrative registers, this study showed that discontinuation of first-line SC-TNFi in patients with IA was associated with increased total direct and indirect costs. Non-persistent patients increased their total aggregated direct and indirect costs by $\$ 1359$ from the 12-month period before to the 12-month period after treatment discontinuation $(\$ 20,802$ vs. $\$ 22,161)$. Conversely, persistent patients significantly lowered their total direct and indirect costs by $\$ 3135$ ( $\$ 16,600$ vs. $\$ 13,465)$ between the same periods. This widening gap in total costs between the patient groups was mainly driven by higher costs related to HCRU and sick leave during the 12-month period following treatment discontinuation in non-persistent compared to persistent patients (\$6961 vs. $\$ 3599$ for HCRU costs and $\$ 7779$ vs. $\$ 3571$ for sick leave costs). While non-persistent patients increased their HCRU costs and maintained their costs because of sick leave from the preindex period, persistent patients significantly decreased these costs over the same period. The findings regarding overall costs were robust in sensitivity analyses on unmeasured confounding and methods to model costs and quantity uncertainty. Disentangled into cost items, the overall decrease in HCRU costs in persistent patients was driven by a significant decrease in costs of outpatient care, whereas the increase in HRCU costs in non-persistent patients was mainly attributable to rising inpatient costs.

It is notable that the diverging cost trajectories for non-persistent and persistent patients were initiated before the index date and that non-persistent patients increased their HCRU costs considerably 1 to 2 months before treatment discontinuation. The increase in HCRU costs in non-persistent patients in the present study concurs with the findings of a study in skin psoriasis with similar design [26], where patients failing biologic treatment were shown to increase the number of physician visits prior to treatment discontinuation. Hence, if the increase in HCRU costs seen in non-persistent patients just prior to treatment discontinuation is attributable to treatment discontinuation, the costs associated with non-persistence in the current study may potentially be underestimated, although a regression-to-the-mean effect, where HRCU costs may peak around treatment discontinuation (when patients' disease status is often at its worst), might also be expected.

Our findings are also consistent with a number of studies [11-13, 27-38] conducted in varying settings, which have assessed health care costs associated with non-persistence in treatment with biologics in IA indications. While most of them evaluated costs only in RA, a few studies considered other IA indications either additionally [12] or exclusively [33, 37]. Apart from the costs of the biologic therapies, persistence with biologics was consistently reported to be associated with cost offsets in HCRU compared to non-persistence.

In addition to costs of HCRU, Ziegelbauer and colleagues [13] found that non-persistence with SC-TNFis was associated with higher indirect costs in treatment for IA in a cohort of German patients. However, both that German study [13] and the most the other studies investigating this matter [11, 12, 28, 31-38] have evaluated costs associated with non-persistence using claims data, which due to selection bias may limit the generalizability to the general IA population. Our study utilizes population-based registers with good coverage, thus allowing for increased generalizability of our results. Furthermore, most previous studies have evaluated costs over a fixed period from the date of treatment initiation, which may have implications for the assessment of costs and stratification. One such implication is that costs associated with non-persistence may not reflect the costs within proximity of treatment discontinuation for non-persistent patients. Second, a fixed period approach implies that two patients with similar persistence estimates, who discontinue their treatment in close relation to the cut-off (e.g., 11 and 13 months, respectively, where persistence status at 12 months is considered), may be categorized differently. In contrast, the current study assessed costs over a 24-month interval around the index date, defined as the date of treatment 
discontinuation among non-persistent patients and applied to their matched persistent controls. This allowed for capturing of costs related to non-persistence closer to the date of SC-TNFi treatment discontinuation and a more valid stratification of patients into persistent and non-persistent categories. Another strength of the current study is the high degree of quality and completeness of the Swedish NHDRs and MiDAS, which may contribute to the accuracy and reliability of our findings [39, 40]. Moreover, the PDR register used in the study captures $>99 \%$ of all dispensed SC-TNFi prescriptions in Sweden [16].

A limitation of our study is the absence of clinical data, which would enable potential identification of clinical drivers of SC-TNFi treatment discontinuation, given that administrative registers largely lack information to investigate such effects. Furthermore, while PSM was implemented, it should be noted that residual confounding may still exist as data on important clinical factors that might have an impact on the presented cost estimates are lacking (e.g., information on disease activity/severity is lacking and may therefore be distributed unequally across persistent and nonpersistent patients). Additionally, although the present study considers the cost of primary care, this restricts our analysis to only a specific subset of the Swedish IA population residing in Västra Götaland County for which such data were available, thereby potentially limiting the generalizability of our results to other settings. While this is a key limitation, it is noteworthy that this is Sweden's second largest county (constituting $17 \%$ of the Swedish population) and that characteristics of eligible IA patients identified correspond very well to the eligible IA patients in the full Swedish IA population, published in our previous study that used the same data sources and patient eligibility criteria [41]. Moreover, the results of our analyses are congruent with those presented for the full Swedish IA population [41], which excluded the costs of outpatient care provided by non-doctors, primary care and employee absenteeism. Although the current study included these additional cost items, it should be highlighted that other cost components, which may be associated with IA, were either partially missing or lacking completely in the utilized data sources. Notably, our study did not consider costs of informal care and sources of direct non-health care costs such as private investments, community services and transportation. As per the findings reported by Jacobsson et al. [42], while community services and transportation may have potentially impacted the total direct costs in a considerable manner, other missing elements such as informal care would likely have contributed only marginally to the overall direct costs. The current study also lacked the ability to capture out-of-pocket expenditures in IA. However, considering the ceiling of SEK 2250 (USD 260) and SEK 1100 (USD 127) in 2018 on out-of-pocket expenses for prescription drugs and medical consultations, respectively, we anticipate these costs to be of minor impact. Furthermore, sick leave episodes $\leq 14$ days were generally not included in MiDAS unless they appeared within 4 days of a previous episode. However, a Swedish study found that only $2 \%$ of sick leave episodes are 8-14 days in RA patients [43] and a Finnish report demonstrated only $3 \%$ of sick leave episodes in RA to be $<10$ days, making up only $0.2 \%$ of total days [44], suggesting that underestimation is likely to be of minor importance. Finally, viewed internationally the Swedish social insurance system is generous, which potentially limits generalizability to other countries.

There is considerable scope for future research in this field. Linking administrative databases to clinical registers could offer valuable insights into the clinical aspects of SC-TNFi therapy in IA, including the associated adverse events, clinical outcomes and medical reasons for treatment discontinuation. Furthermore, more studies examining the full range of costs associated with IA, including primary care and indirect costs, are warranted in settings where such data are recorded and available on a larger, nationwide scale.

\section{CONCLUSION}

Among biologic-naïve patients treated with SCTNFis for IA, non-persistent patients 
significantly increased their costs pertaining to HCRU from the year before to the year after treatment discontinuation. Conversely, persistent patients significantly decreased their costs pertaining to HCRU and sick leave over the same time period, yielding an aggregated cost burden that was almost $40 \%$ lower for treatment-persistent compared to non-treatmentpersistent patients during the year after discontinuation of SC-TNFis. This highlights the impact of treatment persistence from an economic point of view, adding further aspects to the clinical perspective.

\section{ACKNOWLEDGEMENTS}

Funding. Sponsorship for this study and Rapid Service Fee were funded by Merck Sharp \& Dohme Corp., a subsidiary of Merck \& Co., Inc., Kenilworth, NJ, USA.

Authorship. All named authors meet the International Committee of Medical Journal Editors (ICMJE) criteria for authorship for this article, take responsibility for the integrity of the work as a whole and have given their approval for this version to be published.

Author Contributions. All named authors contributed to the concept and design of this study. Johan Dalén performed the statistical analysis, Johan Dalén and Anushri Chitkara summarized the results, Johan Dalén, Anushri Chitkara and Axel Svedbom drafted the manuscript. All named authors revised and approved the manuscript prior to submission.

Disclosures. Zaina P. Qureshi, Amy Puenpatom and Christopher M. Black are employees of Merck Sharp \& Dohme Corp., a subsidiary of Merck \& Co., Inc., Kenilworth, NJ, USA, and hold stock and options in Merck \& Co., Inc., Kenilworth, NJ, USA. Johan Dalén, Anushri Chitkara, and Axel Svedbom are employees of ICON plc. ICON plc were paid consultants to Merck \& Co., Inc., Kenilworth, NJ, USA, in conjunction with the development of this manuscript. ICON plc has received funding from several pharmaceutical companies involved in the marketing products for treatment of inflammatory arthritis. Tor Olofsson is a specialist in rheumatology at Skåne University Hospital and has performed consulting tasks for Merck Sharp \& Dohme related to the present work and for Eli Lilly, unrelated to the present work.

Compliance with Ethics Guidelines. Due to the retrospective non-interventional study design and the applicable laws and regulations pertaining to the utilized data sources, informed consent and participant consent were not applicable. The study was performed in accordance with the Helsinki Declaration and, prior to initiation, ethics approval was granted by the Regional Ethical Review Board in Stockholm, Sweden (approval number 2019-02774).

Data Availability. The datasets generated during and/or analyzed during the current study are not publicly available due to applicable laws and regulations pertaining to Swedish administrative and clinical data available for research.

Open Access. This article is licensed under a Creative Commons Attribution-NonCommercial 4.0 International License, which permits any non-commercial use, sharing, adaptation, distribution and reproduction in any medium or format, as long as you give appropriate credit to the original author(s) and the source, provide a link to the Creative Commons licence, and indicate if changes were made. The images or other third party material in this article are included in the article's Creative Commons licence, unless indicated otherwise in a credit line to the material. If material is not included in the article's Creative Commons licence and your intended use is not permitted by statutory regulation or exceeds the permitted use, you will need to obtain permission directly from the copyright holder. To view a copy of this licence, visit http:// creativecommons.org/licenses/by-nc/4.0/. 


\section{REFERENCES}

1. Handout on Health: Rheumatoid Arthritis. National Institute of Arthritis and Musculoskeletal and Skin Diseases; 2014.

2. Lipton S, Deodhar A. The new ASAS classification criteria for axial and peripheral spondyloarthritis. Int J Clin Rheumatol. 2012;6(7):675-82.

3. Braun J, van den Berg R, Baraliakos X, Boehm H, Burgos-Vargas R, Collantes-Estevez E, et al. 2010 update of the ASAS/EULAR recommendations for the management of ankylosing spondylitis. Ann Rheum Dis. 2011;70(6):896-904.

4. Gossec L, Smolen JS, Gaujoux-Viala C, Ash Z, Marzo-Ortega $H$, van der Heijde D, et al. European League Against Rheumatism recommendations for the management of psoriatic arthritis with pharmacological therapies. Ann Rheum Dis. 2012;71(1): 4-12.

5. Pincus T, Callahan LF. What is the natural history of rheumatoid arthritis? Rheum Dis Clin North Am. 1993;19(1):123-51.

6. Stevens SR, Chang TH. History of development of TNF inhibitors. TNF-alpha inhibitors. Springer; 2006. p. 9-22.

7. Aletaha D, Smolen JS. Effectiveness profiles and dose dependent retention of traditional disease modifying antirheumatic drugs for rheumatoid arthritis. An observational study. J Rheumatol. 2002;29(8):1631-8.

8. Fries J. Effectiveness and toxicity considerations in outcome directed therapy in rheumatoid arthritis. J Rheumatol Suppl. 1996;44:102-6.

9. Pincus T, Marcum S, Callahan L. Longterm drug therapy for rheumatoid arthritis in seven rheumatology private practices: II. Second line drugs and prednisone. J Rheumatol. 1992;19(12):1885-94.

10. Dalen J, Svedbom A, Black CM, Lyu R, Ding Q, Sajjan S, et al. Treatment persistence among patients with immune-mediated rheumatic disease newly treated with subcutaneous TNF-alpha inhibitors and costs associated with non-persistence. Rheumatol Int. 2016;36(7):987-95.

11. Tang B, Rahman M, Waters HC, Callegari P. Treatment persistence with adalimumab, etanercept, or infliximab in combination with methotrexate and the effects on health care costs in patients with rheumatoid arthritis. Clin Ther. 2008;30(7): 1375-84.
12. Belhassen M, Tubach F, Hudry C, Woronoff-Lemsi M, Levy-Bachelot L, Van Ganse E, et al. Impact of persistence with tumour necrosis factor inhibitors on healthcare resource utilization and costs in chronic inflammatory joint diseases. Brit J Clin Pharmacol. 2020;87:163-77.

13. Ziegelbauer K, Kostev K, Hubinger M, Dombrowski $\mathrm{S}$, Friedrichs M, Friedel H, et al. The impact of nonpersistence on the direct and indirect costs in patients treated with subcutaneous tumour necrosis factor-alpha inhibitors in Germany. Rheumatology (UK). 2018;57(7):1276-81.

14. Forsblad-d'Elia H, Jacobsson LT, Feltelius N, Husmark T, Szentpetery A, Wallman JK, et al. Riktlinjer för läkemedelsbehandling vid axial spondylartrit och psoriasisartrit 2019. Svensk Reumatologisk Förening; 2019.

15. Gjertsson I, Lampa J, Svärd A, Södergren A, Turesson C. Riktlinjer för läkemedelsbehandling vid reumatoid artrit. Svensk Reumatologisk Förening; 2019.

16. Welfare NBoHa. Quality declaration, statistics on pharmaceuticals for 2018. 2019.

17. Cramer JA, Roy A, Burrell A, Fairchild CJ, Fuldeore MJ, Ollendorf DA, et al. Medication compliance and persistence: terminology and definitions. Value Health. 2008;11(1):44-7.

18. Statistics Sweden. Genomsnittlig månadslön, lön i fasta priser och lönespridning efter utbildningsnivå SUN 2000 och kön. Âr 1991-2019, [updated 13 Jan 2021. Available from: https://www.scb.se/hittastatistik/sverige-i-siffror/utbildning-jobb-ochpengar/medelloner-i-sverige/.

19. Rosenbaum PR, Rubin DB. The central role of the propensity score in observational studies for causal effects. Biometrika. 1983;70(1):41-55.

20. Austin PC. Balance diagnostics for comparing the distribution of baseline covariates between treatment groups in propensity-score matched samples. Stat Med. 2009;28(25):3083-107.

21. Ho DE, Imai K, King G, Stuart EA. Matching as nonparametric preprocessing for reducing model dependence in parametric causal inference. Polit Anal. 2007;15(3):199-236.

22. Imai K, King G, Stuart EA. Misunderstandings between experimentalists and observationalists about causal inference. Field experiments and their critics. Yale: Yale University Press; 2014. p. 196-227.

23. Desgagné A, Castilloux A-M, Angers J-F, Le Lorier J. The use of the bootstrap statistical method for the 
pharmacoeconomic cost analysis of skewed data. Pharmacoeconomics. 1998;13(5):487-97.

24. Mathur MB, Ding P, Riddell CA, VanderWeele TJ. Website and $\mathrm{R}$ package for computing E-values. Epidemiology. 2018;29(5):e45.

25. VanderWeele TJ, Ding P. Sensitivity analysis in observational research: introducing the E-value. Ann Intern Med. 2017;167(4):268-74.

26. Svedbom A, Dalén J, Mamolo C, Cappelleri JC, Mallbris L, Petersson IF, et al. Economic burden of psoriasis and potential cost offsets with biologic treatment: a Swedish Register Analysis. Acta Derm Venereol. 2016;96(5):651-7.

27. Tarallo M, Onishchenko K, Alexopoulos ST. Costs associated with non-medical switching from originator to biosimilar etanercept in patients with rheumatoid arthritis in the UK. J Med Econ. 2019;22(11):1162-70.

28. Sruamsiri R, Kameda H, Mahlich J. Persistence with biological disease-modifying antirheumatic drugs and its associated resource utilization and costs. Drugs-real World Outcomes. 2018;5(3):169-79.

29. Degli Esposti L, Favalli EG, Sangiorgi D, Di Turi R, Farina G, Gambera M, et al. Persistence, switch rates, drug consumption and costs of biological treatment of rheumatoid arthritis: an observational study in Italy. ClinicoEcon Outcomes Res CEOR. 2017;9:9.

30. Cannon GW, DuVall SL, Haroldsen CL, Caplan L, Curtis JR, Michaud K, et al. Clinical outcomes and biologic costs of switching between tumor necrosis factor inhibitors in US veterans with rheumatoid arthritis. Adv Ther. 2016;33(8):1347-59.

31. Meissner B, Trivedi D, You M, Rosenblatt L. Switching of biologic disease modifying anti-rheumatic drugs in patients with rheumatoid arthritis in a real world setting. J Med Econ. 2014;17(4):259-65.

32. Rashid N, Lin AT, Aranda G Jr, Lin KJ, Guerrero VN, Nadkarni A, et al. Rates, factors, reasons, and economic impact associated with switching in rheumatoid arthritis patients newly initiated on biologic disease modifying anti-rheumatic drugs in an integrated healthcare system. J Med Econ. 2016;19(6):568-75.

33. Hur P, Kim N, Dai D, Piao OW, Zheng JZ, Yi E. Healthcare cost and utilization associated with biologic treatment patterns among patients with psoriatic arthritis: analyses from a large US Claims database. Drugs-Real World Outcomes. 2020, pp 1-10.
34. Shahabi A, Shafrin J, Zhao L, Green S, Curtice T, Marshall A, et al. The economic burden of switching targeted disease-modifying anti-rheumatic drugs among rheumatoid arthritis patients. J Med Econ. 2019;22(4):350-8.

35. Vanderpoel J, Tkacz J, Brady BL, Ellis L. Health care resource utilization and costs associated with switching biologics in rheumatoid arthritis. Clin Ther. 2019;41(6):1080-9.e5.

36. Gu T, Mutebi A, Stolshek BS, Tan H. Cost of biologic treatment persistence or switching in rheumatoid arthritis. Am J Managed Care. 2018;24:SP338-435.

37. Yi E, Dai D, Piao OW, Zheng JZ, Park Y. Health care utilization and cost associated with switching biologics within the first year of biologic treatment initiation among patients with ankylosing spondylitis. J Manag Care Spec Pharm. 2021;27(1): 27-36.

38. Wang H, Wang Y, Michael T, Hopkins B, Bodoria M, Sondhi M, et al. PMS9 biologic therapy patterns and associated costs in rheumatoid arthritis patients who initiated a tumor necrosis factor antagonist over two years. Value Health. 2012;15(4):A35.

39. Ludvigsson JF, Andersson E, Ekbom A, Feychting M, Kim JL, Reuterwall C, et al. External review and validation of the Swedish national inpatient register. BMC Public Health. 2011;11:450.

40. Wettermark B, Hammar N, Fored CM, Leimanis A, Otterblad Olausson P, Bergman U, et al. The new Swedish Prescribed Drug Register-opportunities for pharmacoepidemiological research and experience from the first six months. Pharmacoepidemiol Drug Saf. 2007;16(7):726-35.

41. Dalen J, Luttropp K, Olofsson T, Black C, Puenpatom A, editors. Cost of non-persistence in the treatment with subcutaneous tumor necrosis factoralpha inhibitors of inflammatory arthritis: a propensity score matching approach. Arthritis \& Rheumatology; 2020: Wiley 111 River St, Hoboken 07030-5774, NJ USA.

42. Jacobsson L, Lindroth Y, Marsal L, Juran E, Bergström U, Kobelt G. Rheumatoid arthritis: what does it cost and what factors are driving those costs? Results of a survey in a community-derived population in Malmö Sweden. Scand J Rheumatol. 2007;36(3):179-83.

43. Hubertsson J, Englund $\mathrm{M}$, Hallgårde U, Lidwall U, Löfvendahl S, Petersson IF. Sick leave patterns in common musculoskeletal disorders-a study of doctor prescribed sick leave. BMC Musculoskelet Disord. 2014;15(1):1-9. 
44. Puolakka K, Kautiainen H, Pekurinen M, Möttönen T, Hannonen P, Korpela M, et al. Monetary value of lost productivity over a five year follow up in early rheumatoid arthritis estimated on the basis of official register data on patients' sickness absence and gross income: experience from the FIN-RACo trial. Ann Rheum Dis. 2006;65(7):899-904. 